Article type: Full Paper

\title{
A New Method for Determining the Composition of Core-Shell Nanoparticles via Dual- EDX+EELS Spectrum Imaging
}

David Rossouw ${ }^{1 *}$, Benjamin R. Knappett ${ }^{2}$, Andrew E. H. Wheatley², Paul A. Midgley ${ }^{1}$.

1. Department of Materials Science and Metallurgy, University of Cambridge, 27 Charles Babbage Road, Cambridge CB3 OFS, United Kingdom.

2. Department of Chemistry, University of Cambridge, Lensfield Road, Cambridge CB2 1EW, United Kingdom.

Keywords: nanoparticles, core-shell, EDX, EELS, ICA

Simultaneously acquired micro-analytical x-ray and electron energy loss signals were obtained from a bimetallic core-shell nanoparticle system $\left(\mathrm{FePt} @ \mathrm{Fe}_{3} \mathrm{O}_{4}\right)$. The signals were decomposed using independent component analysis and the extracted components were used to separately quantify the composition of the spatially overlapping core and shell phases in the nano-heterostructure. The utilization of the complementary strengths of energy dispersive $\mathrm{x}-$ ray and electron energy-loss spectroscopy microanalysis has enabled the quantification of both light and heavy elements in a single spectrum image acquisition.

\section{Introduction}

The scanning transmission electron microscope (STEM) has had several upgrades to its analytical capabilities in recent years. The latest generation of STEMs are typically equipped with energy dispersive X-ray (EDX) systems with much improved signal collection efficiencies over the conventional systems fitted to instruments assembled in the past decade ${ }^{[1,2]}$. Modern STEMs are also fitted with ultra-fast electron energy loss spectroscopy (EELS) spectrometers ${ }^{[3]}$. These contemporary systems have reduced the acquisition time and electron dose required to acquire high spatial resolution two-dimensional (2D) elemental maps with good signal-to-noise ratios. The location of the EDX, EELS and imaging detectors in a typical STEM system permits the simultaneous acquisition of signal outputs from these detectors. 
Despite the obvious advantage of acquiring multiple signals for a more complete characterization of a material, examples of simultaneous EELS and EDX acquisitions in the literature are few, with some limitations in hardware, software, acquisition and synchronization remaining. In 2014, Kothleitner and colleagues acquired simultaneous lowloss and high-loss EELS and EDX signals and were able to demonstrate assumption-free compositional analysis ${ }^{[4]}$. Dual EDX+EELS analysis has also been applied recently to electron tomography to obtain 3D elemental reconstructions ${ }^{[5]}$.

EELS and EDX can be viewed as complementary microanalysis techniques. EDX has a wide energy range (typically $0-20 \mathrm{keV}$ ) allowing for the detection of nearly all the elements in the periodic table in a single acquisition. However, low energy X-rays from light elements are subject to strong absorption before detection in both the sample and detector. This is in spite of modern windowless detector designs minimizing x-ray absorption in the detector and offering improved x-ray visibility at low energies. EELS offers superior energy resolution, enabling access to chemical bonding information, but over a much more limited and lower energy range (typically less than $2-3 \mathrm{keV}$ ). As a result, X-ray quantification is typically performed for heavy elements and EELS quantification for light elements. One potential benefit of combining EDX and EELS is, therefore, access to both light and heavy element quantification from a single acquisition.

The capacity to rapidly acquire large EDX and EELS spectrum images with modern hardware has also pushed the need for efficient methods to analyze big datasets ${ }^{[6]}$. Multivariate statistical techniques, including principal component analysis (PCA) and independent component analysis (ICA) ${ }^{[7]}$, can be used to recast raw spectrum image data onto a new set of additive basis factors, each with an associated loading coefficient. Large datasets can often be well represented by a just a few basis factors, rather than thousands if one considers each 
pixel in a large spectrum image separately. PCA decomposes a dataset into a set of component factors. The first factor represents the maximum variance in the raw data, and each succeeding factor the next highest variance orthogonal to the preceding factors. On the other hand, ICA decomposes a dataset into statistically independent components, defined as maximally non-Gaussian in their joint distribution. A common analogy of ICA is the 'cocktail party problem', in which the challenge is to separate the sounds of individual speakers from the mixed conversations recorded by microphones at different locations in a room. In the case of spectrum imaging, each $(\mathrm{x}, \mathrm{y})$ pixel can be thought of as a microphone, and the signal axis (E) as the recorded sound amplitude. ICA has been applied to EDX ${ }^{[8,9]}$ and EELS ${ }^{[10,11]}$ spectrum images. More recently, the components of ICA have been used to find the composition of the bimetallic core in a core-shell nanoparticle system ${ }^{[12]}$ and the composition of gamma' precipitates in a nickel-based superalloy ${ }^{[13]}$. Here, we apply PCA and ICA methods to a dual EDX+EELS dataset, enabling the measurement of both core and shell compositions in core-shell heterostructures.

\section{Materials and Methods}

A solution of magnetic core-shell nano-particles, comprising FePt bimetallic cores and iron oxide shells, was drop-cast onto a $3 \mathrm{~mm}$ holey carbon copper grid prior to characterization by electron microscopy. EDX and EELS data were acquired using an FEI Osiris TEM/STEM equipped with a high brightness Schottky X-FEG gun, a Super-X EDX system and a Gatan Enfinium EELS spectrometer. High resolution STEM images were obtained separately on an aberration corrected FEI Titan cubed TEM. The Osiris TEM was operated at $200 \mathrm{kV}$ and the native TIA software was used to acquire the dual EDX+EELS spectrum images, in which the raster beam scan was coordinated with the simultaneous collection of HAADF, EDX and EELS signals (Fig. 1). 


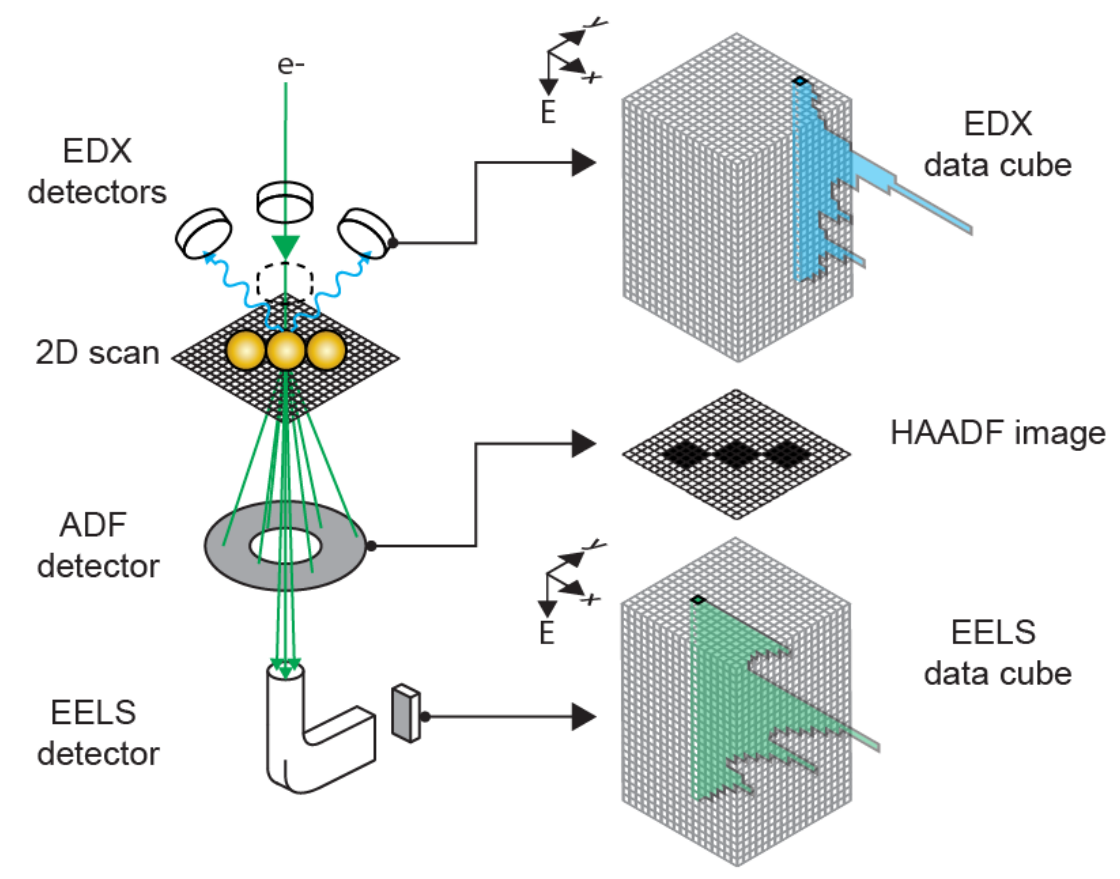

Figure 1. A schematic of the simultaneous acquisition of EDX and EELS datasets.

Data post-processing and EDX and EELS quantification was performed using the HyperSpy software package ${ }^{[14]}$ and Digital Micrograph. PCA and ICA decompositions were also performed using the FASTICA algorithm ${ }^{[15]}$ as implemented in Scikit learn ${ }^{[16]}$ and accessed in the machine learning package of HyperSpy.

\section{Results}

\subsection{STEM Imaging}

Figure 2 displays HAADF-STEM images of a (a) small nanoparticle cluster and (b) an isolated nanoparticle. The higher brightness of the particle core relative to the shell is consistent with the expected FePt@Fe3O4 core-shell composition based on the findings of a previous study ${ }^{[17]}$. The atomically resolved images also indicate that the crystalline metallic core is surrounded by a continuous oxide layer that lacks long-range order and appears partially amorphous in character. 
(a)

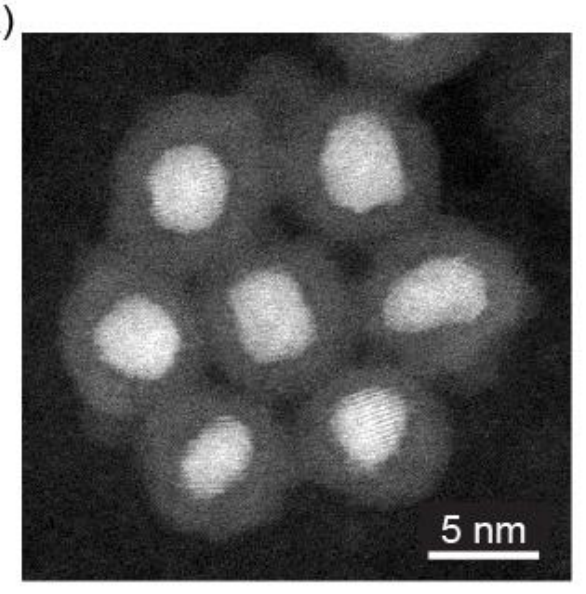

(b)

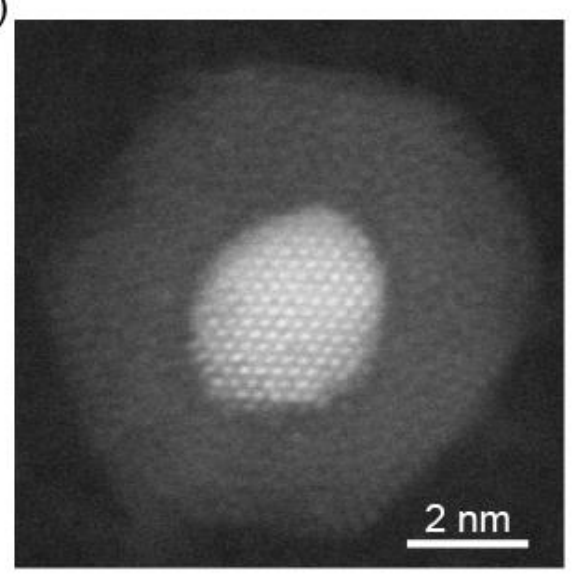

Figure 2. HAADF-STEM imaging of FePt@iron oxide nanoparticles. (a) HR-STEM image of a particle cluster. (b) Enlarged and frame averaged image of an isolated particle.

\subsection{Independent Component Analysis}

In an attempt to to achieve optimum magnetic properties, a bimetallic core composition of 50/50 FePt was targeted synthetically. However, due to the spatial overlap of the core and the shell in projection, it is challenging to quantify the composition of these phases using traditional methods because the signals from each phase will be mixed upon detection. In the present case, we acquire both EDX and EELS signals from a representative particle cluster and utilize multivariate statistical methods, including PCA and ICA, to separate the mixed signals originating from the particle cores and shells. These separated signals, or components, are subsequently analyzed and quantified to find the compositions of particle cores and shells.

\subsection{1. $E D X-P C A$}

Figure 3 displays PCA results of the EDX spectrum image obtained from a region enclosing a representative cluster of particles (a,i). The spectrum image was acquired with a $0.7 \mathrm{nA}$ focused probe, sampled at 40x40 pixels with a $1 \mathrm{~nm}$ pixel size and a dwell time of 100 ms/pixel. The scree plot (a,ii) shows that most of the variance in the dataset can be explained 
by three orthogonal principal components (PCs); PC\#0, PC\#1 and PC\#2 (the first five PCs are displayed in the SI). The component factors are plotted in (a) and their respective pixel-wise loading weights are displayed in grey-scale in (b-d). PC\#0 contains peaks from all the elements expected to be present in the region, namely carbon (from the support membrane), oxygen, iron and platinum $(\mathrm{C}, \mathrm{O}, \mathrm{Fe}, \mathrm{Pt})$. A copper $(\mathrm{Cu})$ peak is also detected and is assumed to be spurious in origin, likely coming from the supporting copper grid bars. The spatial loading of PC\#0 matches the HAADF-STEM signal and is positively valued over the particle cores and shells. PC\#1 contains a strong C-K $\square$ peak and negative Pt-L and Pt-M peaks. Spatially, PC\#1 is positively valued in the support region but negatively valued in the particle core regions. Finally, PC\#2 contains a negative C-Ka peak, positive O-Ka and Fe-La peaks, negative Pt-L and Pt-M peaks, and positive Fe-Ka and Fe-Kb peaks. The PC\#2 loading map is positively valued over the particle shells, close to zero over the support and negatively valued over the particle cores. A summary of the principal component decomposition is provided in Table 1.

(a)

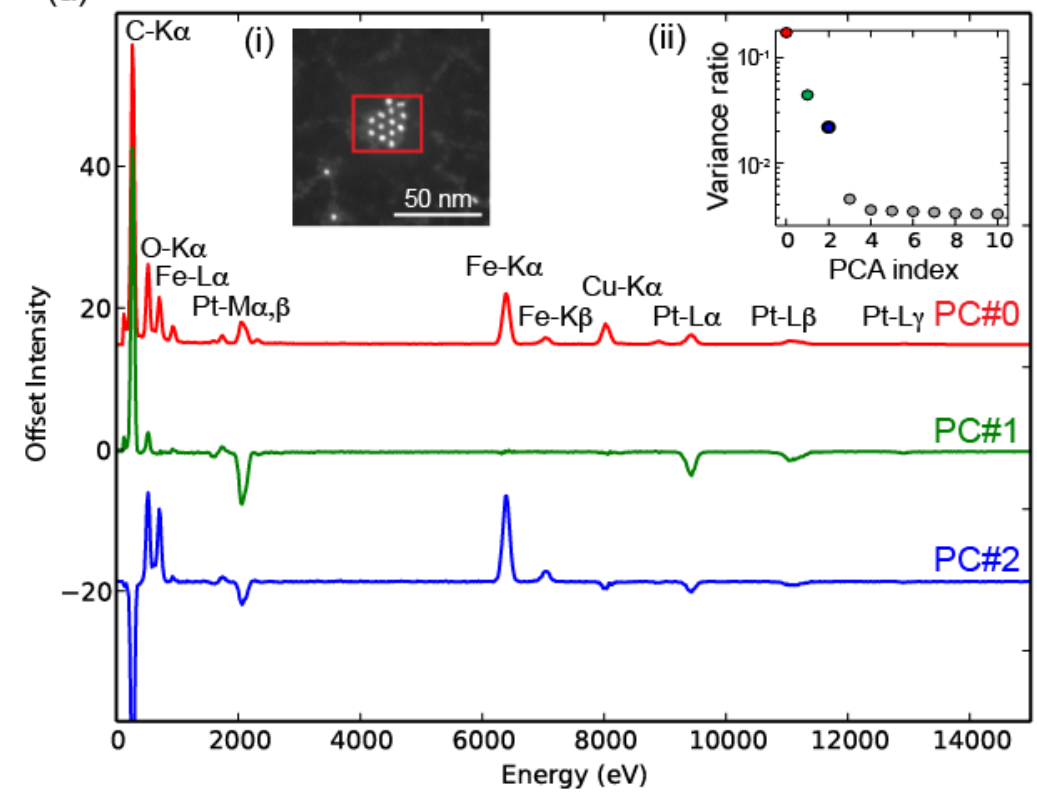

(b)

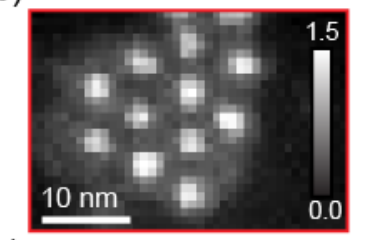

(c)

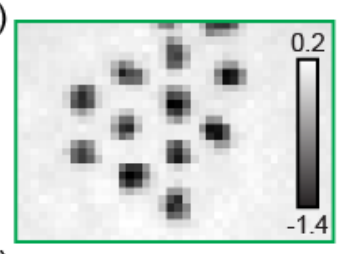

(d)

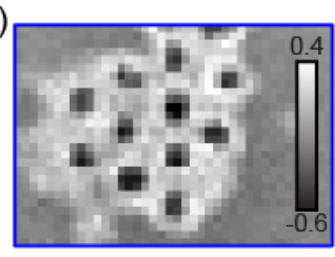

Figure 3. PCA of the EDX spectrum image. (a) The extracted PC factors PC\#0 (red), PC\#1 (green) and PC\#2 (blue) from (i) a region enclosing a nanoparticle cluster (red box). (ii) A 
scree plot of the variance in the dataset. The spatial loadings of (b) PC\#0, (c) PC\#1 and (d) PC\#2.

Table 1. PCA decomposition summary.

\begin{tabular}{|c|c|c|c|c|c|}
\hline \multirow[t]{2}{*}{ PC\# } & \multicolumn{2}{|c|}{ Peaks present } & \multicolumn{3}{|c|}{ Loading polarity } \\
\hline & Positive & Negative & Core & Shell & Support \\
\hline 0 & 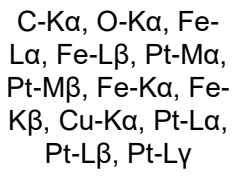 & None & Positive & Positive & Zero \\
\hline 1 & $\mathrm{C}-\mathrm{K} \alpha, \mathrm{O}-\mathrm{K} \alpha$ & $\begin{array}{l}\text { Pt-M } \alpha, \text { Pt-M } \beta, \text { Pt- } \\
\text { L } \alpha, \text { Pt-L } \beta, \text { Pt-L } \gamma\end{array}$ & Negative & Positive & Positive \\
\hline 2 & $\begin{array}{l}\mathrm{O}-\mathrm{K} \alpha, \mathrm{Fe}-\mathrm{L} \alpha, \mathrm{Fe}- \\
\mathrm{L} \beta, \mathrm{Fe}-\mathrm{K} \alpha, \mathrm{Fe}-\mathrm{K} \beta\end{array}$ & 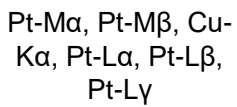 & Negative & Positive & Zero \\
\hline
\end{tabular}

While the PCA decomposition shows that just three principal components are sufficient to explain most of the variance in the spectrum image, the components themselves, both in spectral and spatial form, are difficult to interpret physically by virtue of the presence of negative factor peaks and negative loading values.

\subsubsection{EDX-ICA}

Given the difficulty of interpreting PCA, we have developed the use an alternative decomposition method, ICA, in an attempt to obtain a clearer picture of the internal data structure. Figure 4 displays a summary of the ICA decomposition of the EDX spectrum image analyzed in the previous Section. From the PCA analysis above, it was found that the spectrum image could be largely explained by three PCs. Thus three independent components (ICs) have been sought by ICA. The extracted IC factors are displayed in Fig. 4(a) and their respective pixel-wise loading weights are displayed in grey-scale in (b-d). 

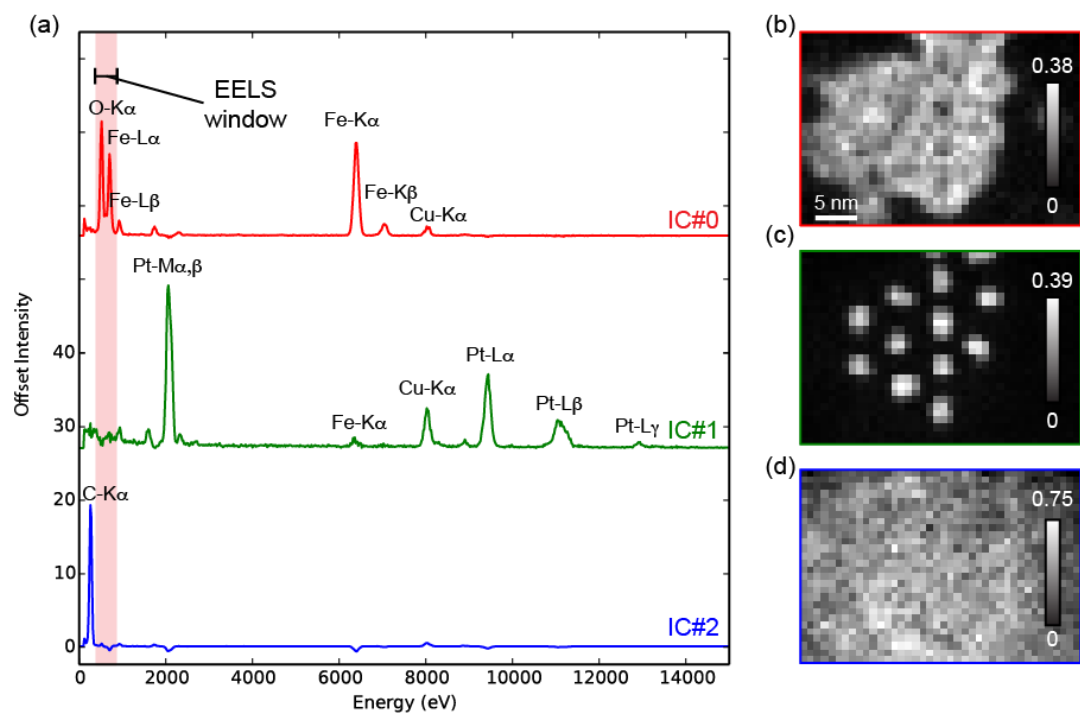

Figure 4. ICA of the EDX spectrum image. (a) The extracted IC factors IC\#0 (red), IC\#1 (green) and IC\#2 (blue). The spatial loadings of (b) IC\#0, (c) IC\#1 and (d) IC\#2.

Most notably, the ICA decomposition does not contain any appreciable negative peaks in the factors or negative weights in the loadings. Based on the X-ray peaks present in each factor and their respective spatial distributions, it appears as though IC\#0 represents the iron oxide shell, IC\#1 the Pt-rich bimetallic cores and IC\#2 the carbon support. A summary of the X-ray peaks present and the loadings is provided in Table 2.

Table 2. ICA decomposition summary.

\begin{tabular}{|c|c|c|c|c|c|}
\hline \multirow[t]{2}{*}{ IC\# } & \multicolumn{2}{|c|}{ Peaks present } & \multicolumn{3}{|c|}{ Loading polarity } \\
\hline & Positive & Negative & Core & Shell & Support \\
\hline 0 & $\begin{array}{c}\text { O-Ka Fe-La, Fe- } \\
\text { L } \beta, \mathrm{Fe}-\mathrm{Ka}, \mathrm{Fe}-\mathrm{L} \beta \\
\mathrm{Cu}-\mathrm{K \alpha}\end{array}$ & None & $\begin{array}{l}\text { Positive } \\
\text { (reduced) }\end{array}$ & Positive & Zero \\
\hline 1 & 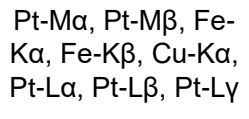 & None & Positive & Zero & Zero \\
\hline 2 & $\mathrm{C}-\mathrm{K} \alpha$ & None & Absent & Absent & Positive \\
\hline
\end{tabular}

\subsubsection{EELS-ICA}

We now turn to analysis of the EELS spectrum image that was acquired simultaneously with the EDX signal. Given the success of the ICA applied to the EDX data cube, we applied the 
same decomposition method to the EELS dataset. Figure 5 displays a summary of the resulting ICA decomposition. As with the EDX spectrum image, the total variance in the EELS dataset can be largely explained by three PCs (a, i). Thus, 3 components were selected for ICA (the first five PCs are displayed in the SI). The extracted IC factors are shown in Fig. 5(a) and their respective spatial loadings in (b-d). The IC factors strongly resemble typical EELS core-loss spectral shapes. We observe an exponentially decaying 'background' in each of the components. IC\#0 and IC\#1 also feature saw-tooth edges containing fine structural oscillations superimposed on the background signal. The location of the first and second edges in IC\#0 coincide with the expected location of O-K and Fe-L23 ionization edges at 532 $\mathrm{eV}$ and $702 \mathrm{eV}$ respectively. The IC\#0 loading is concentrated over the particle shells. The location of the first and second edges in IC\#1 coincide with the expected ionization edges of Pt-N23 and Fe-L23, and spatially it maps to the location of the particle cores. IC\#2 contains no appreciable features above the background. It maps over the entire region, with increased intensity at the edges of the particle cluster. It is likely to represent the extended tail of the lower energy carbon edge at $284 \mathrm{eV}$, which lies outside of the EELS detection window, and is attributed to grid material. 
(a)

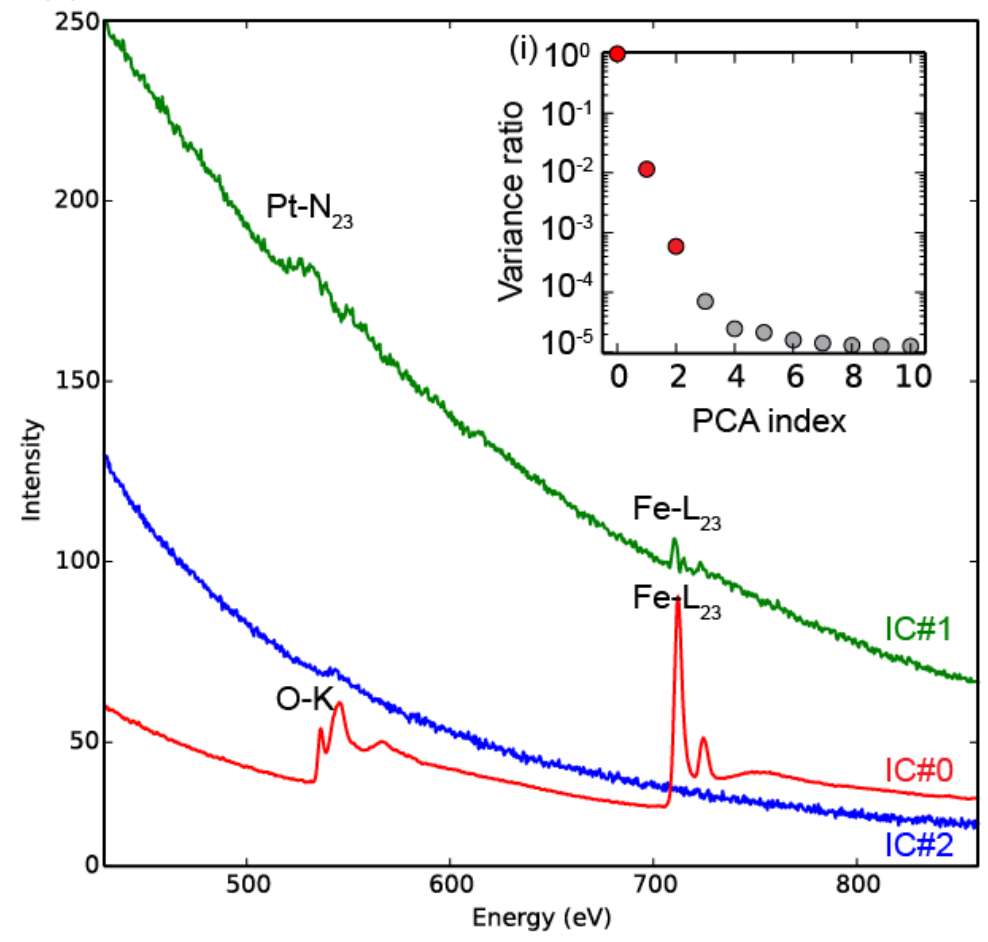

(b)

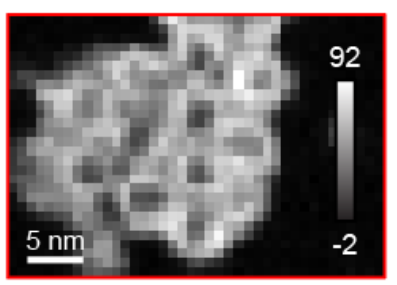

(c)

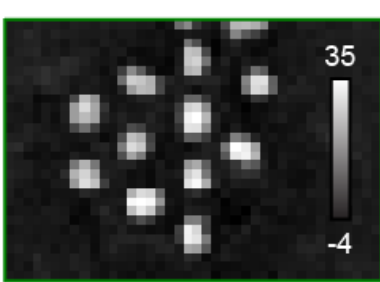

(d)

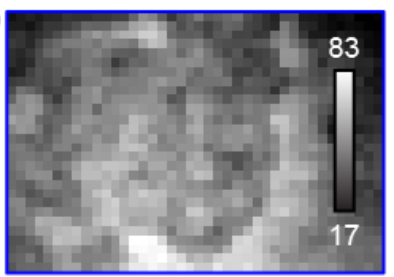

Figure 5. ICA of the EELS spectrum image. (a) The extracted IC factors IC\#0 (red), IC\#1 (green) and IC\#2 (blue) and a scree plot of the dataset (i). The spatial loadings of (b) IC\#0, (c) IC\#1 and (d) IC\#2.

The fine structure in the O-K and Fe-L23 edges observed in IC\#0 and the Pt-N23 and Fe-L23 edges in IC\#1 are in excellent agreement with reference spectra ${ }^{[18]}$.

\subsection{Quantification}

Having obtained what appear to be physically interpretable decompositions of the EDX and EELS spectrum images obtained simultaneously from the representative particle cluster, we have now attempted to find the compositions of the bimetallic particle cores and the phase of the iron oxide shells by quantifying the unmixed component factors obtained by ICA. For the bimetallic cores, ICA has been recently used to successfully extract their composition ${ }^{[12]}$. Here we repeat general the methodology on these core-shell particles, which were produced in the same synthesis, but we now extend the quantification to both EDX and EELS datasets. 


\subsubsection{EDX quantification}

Figure 6 displays the quantification of IC\#0 extracted by ICA from the EDX spectrum image. The Fe-K $\alpha$ and Pt-L $\alpha$ peaks were fitted to Gaussian functions, whose amplitudes, along with standard-less k-values, were used in the Cliff-Lorimer quantification method. A Pt:Fe composition of 85:15 (at.\%) was calculated. This composition lies within one standard deviation (3.3 at\%) of the average FePt bimetallic seed composition measured previously (82.0:18.0 at.\% Pt:Fe) ${ }^{[12]}$.

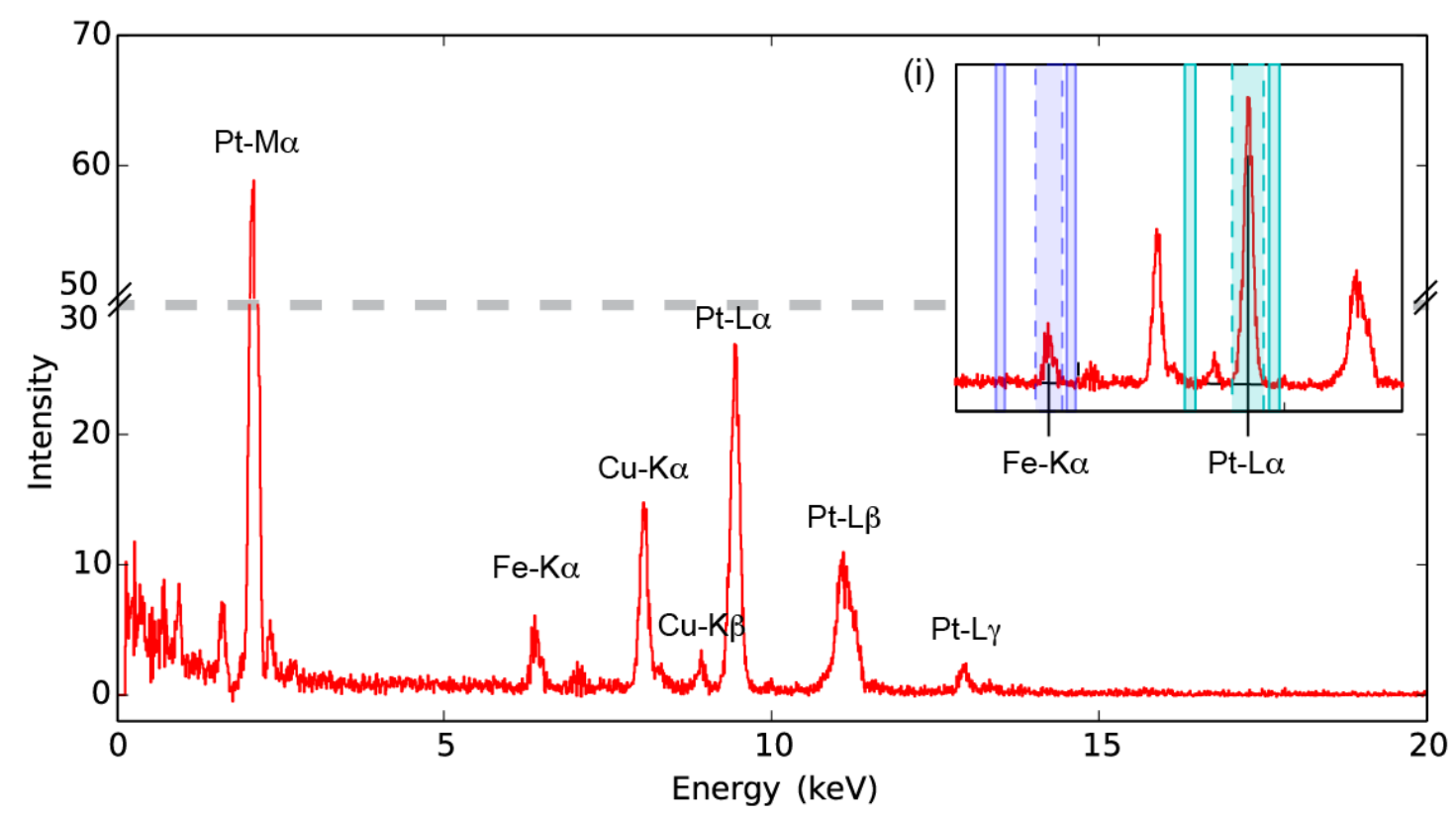

Figure 6. EDX quantification of the IC representing the bimetallic cores (IC\#0). (i) Integrated intensities of the Fe-K $\square$ and Pt-L $\square$ peaks were extracted by background subtraction using a two window method (boxed, solid) prior to Gaussian fitting of the peaks (boxed, dashed).

\subsubsection{EELS quantification}

Figure 7 displays the analysis of energy loss near-edge structures (ELNES) of the O-K and Fe-L23 edges contained in EELS component IC\#0. Good agreement between the shell-only spectrum and component IC\#0 is observed in (a). Previous systematic EELS studies of the 
iron-oxygen system have shown that the oxygen and iron ELNES can be used to determine the nature of the iron-oxide phase, be it $\mathrm{FeO}, \mathrm{Fe} 3 \mathrm{O} 4, \square-\mathrm{Fe} 2 \mathrm{O} 3$ or $\square-\mathrm{Fe} 2 \mathrm{O} 3^{[19,20]}$. For the O$\mathrm{K}$ edge, the intensity of the pre-peak (recorded maximum at $535 \mathrm{eV}$ ) has been observed to increase relative to the dominant peak (maximum at $546 \mathrm{eV}$ ) with increasing oxygen fraction ${ }^{[19]}$. By adopting a similar approach to that described by Colliex and co-workers we fitted the pre-peak and dominant peak in the $\mathrm{O}-\mathrm{K}$ signal to two Gaussian functions and obtained an integrated peak area ratio of $0.16+/-0.02$ (Fig. $7 b$ ). This peak-ratio is uniquely consistent with that measured for $\mathrm{Fe} 3 \mathrm{O} 4{ }^{[19]}$. The peak-ratio error was estimated by recalculation of the area ratio with varying Gaussian fit parameters. Multiple scattering effects are assumed to be negligible for the nanometer-sized particles analyzed.

The Fe-L23 ELNES features two sharp white-line peaks (Fig. 7c). By again following the procedure outlined in ${ }^{[19]}$, we measure a white-line intensity ratio of $4.7+/-0.4$, which is within error of that expected for $\mathrm{FeO}$ and $\mathrm{Fe} 3 \mathrm{O} 4$. $\mathrm{FeO}$ can be ruled out as a candidate structure because it features a substantially reduced O-K pre-peak, contrary to our observations. The larger error estimate for the Fe-L23 white-line ratio here is due to uncertainty in the shape of the double arctan continuum background, which was subtracted from the signal prior to peak fitting (Fig. 7c insert). It should also be noted that an improved fit of the Fe-L23 white lines was obtained by fitting two Gaussians to the L3 peak, and one to the L2 peak. The physical origin of the double Gaussian Fe-L3 fit may be due to crystal field effects, which act to split the degenerate $3 \mathrm{~d}$ orbitals of an isolated Fe cation into multiplet orbital levels in the extended solid, depending on the symmetry of the surrounding ligand structure ${ }^{[21]}$. Based on the ELNES analysis of the O-K and Fe-L23 edges we conclude that the shell is likely Fe3O4. 

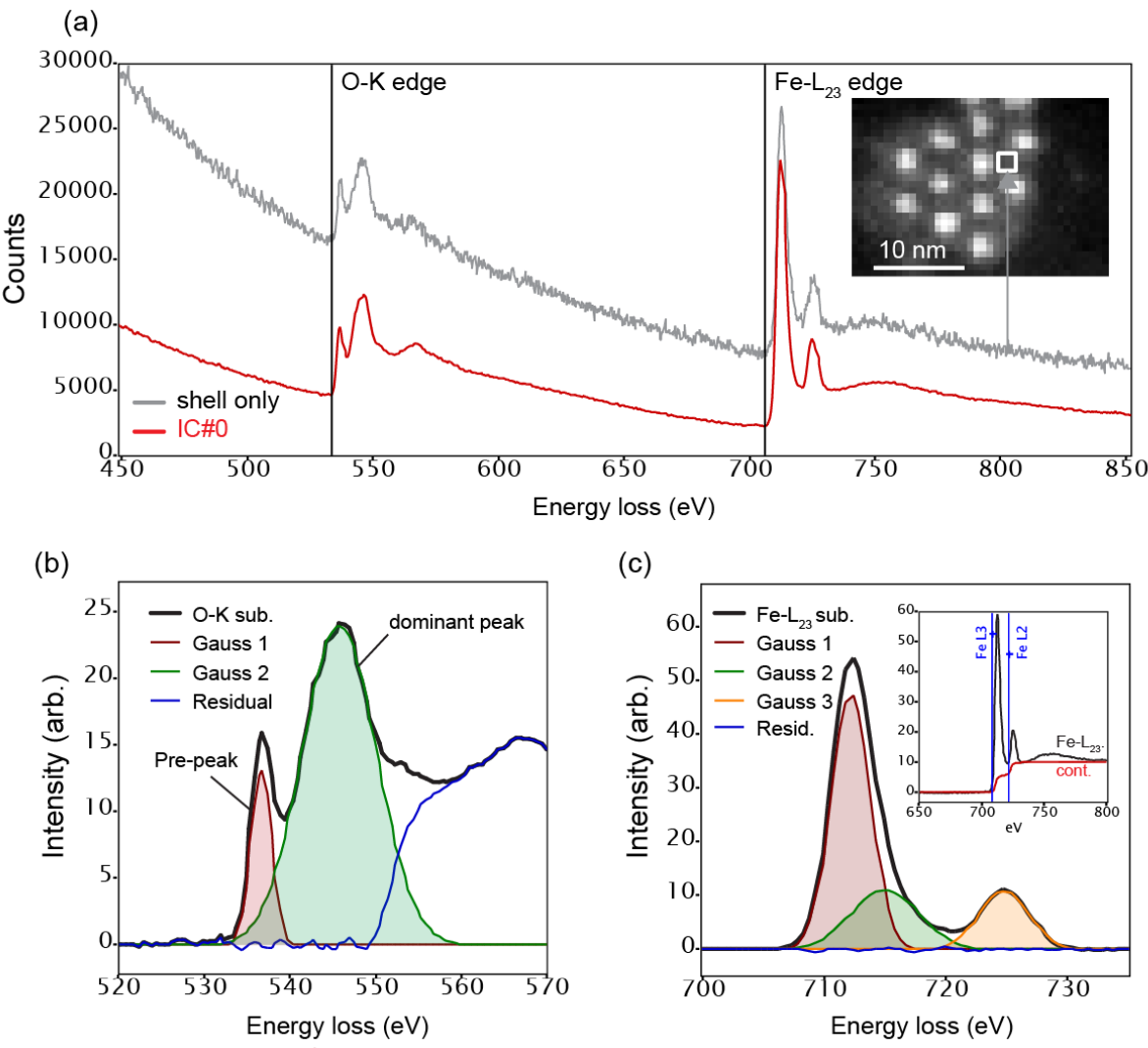

Figure 7. Analysis of the EELS IC representing the particle shells (IC\#0). (a) A comparison of a shell-only spectrum (location shown in insert) with IC\#0. (b) The background subtracted O-K edge (O-K sub.) featuring a Gaussian-fitted pre-peak (red) and dominant peak (green). (c) The background and continuum subtracted Fe-L23 edge (Fe-L23 sub.) featuring Gaussianfitted double white-line peaks L3 (red+green) and L2 (orange). The background-only subtracted edge and double arctan-fitted continuum are shown in the insert.

\section{Discussion}

As detailed above, the variance in both the EDX and EELS spectrum images obtained from a region enclosing a cluster of $\mathrm{FePt} @ \mathrm{Fe} 3 \mathrm{O} 4$ core-shell nanoparticles, was found to be largely explained by three principal components. In addition, the three independent components extracted from each dataset were each found to be representative of the overlapping phases present in the sampled region; the bimetallic core, the iron oxide shell and the carbon support. This consistency in the EDX and EELS decompositions, in addition to the general appearance 
of the component spectra, lent credence to their physical interpretation. Furthermore, the quantification of the EDX ICA core spectrum provided a bimetallic Pt:Fe composition which was in excellent agreement with composition measurements on the bare particle cores reported previously ${ }^{[12]}$. If the spatially overlapping shell is not accounted for when quantifying the EDX spectra extracted from the core regions, conventional standardless CliffLorimer quantification gives an iron-platinum atomic ratio of $\mathrm{Pt}: \mathrm{Fe}=61: 39$ at. $\%$ in the core regions. The additional contribution of iron in the shell to the EDX spectrum biases the bimetallic core quantification, resulting in a higher apparent iron content in the core than that obtained from EDX IC\#0 (Pt:Fe = 85:15 at.\%).

In the compositional analysis of the iron and oxygen-containing particle shells, EDX quantification cannot be reliably used due to the inherently high X-ray absorption of low energy oxygen X-rays by either the sample or the instrumentation. We therefore utilized the simultaneously obtained EELS signals and the ICA decomposition to obtain a more complete characterization of the shell. By calculating peak intensity-ratios for the O-K and Fe-L23 ELNES signals, we determined that the shells were likely Fe3O4. These findings were consistent with similarly obtained measurements on an analogous system ${ }^{[17]}$. The sharp white-line peaks in the Fe-L23 ELNES arise from electron transitions to from the 2p3/2 and 2 p1/2 spin-orbit split levels to unoccupied $3 \mathrm{~d}$ levels ${ }^{[21]}$. They are not included in the HartreeSlater central field model, which assumes elements are in atomic form, and their presence can lead to strong deviations from theoretical scattering cross-sections of up to $50 \%$ for transitionmetal oxides ${ }^{[22]}$, thus compromising scattering cross-section-based quantification. For example, when applied to the EELS shell only and ICA spectra obtained here, Hartree-Slater based quantification yield iron-oxygen ratios (Fe:O) of 31:69 and 33:67 respectively (Fig. S3). Theses ratios differ appreciably from that expected for the $\mathrm{Fe}_{3} \mathrm{O}_{4}$ phase $(\mathrm{Fe}: \mathrm{O}=43: 57)$. It was therefore necessary to adopt the white-line intensity ratio-based approach for this iron oxide system $^{[19]}$. Another advantage of PCA and ICA analysis over conventional spectrum image 
quantification methods lies in the noise reduction of the decomposed dataset. This stems from the rejection of low variance components present in the raw data. In the present context, decomposition of the EELS dataset enabled the Pt-N23 edge to be visible above the noise (Fig. $5 a)$.

\section{Conclusions}

The synergistic use of EDX and EELS, in the form of dual spectrum imaging, complemented by powerful data decomposition methods, enabled the complete composition of bimetallic core-shell nano-heterostructures to be determined from a single beam scan. Here, EDX signals were used to determine the composition of the bimetallic particle cores, and simultaneously acquired EELS signals were used to determine the composition of the particle shells. The capacity to reliably separate such mixed analytical signals from complex heterostructures can also reduce the number of analytical steps that might otherwise be taken en-route to the synthesis of nano-heterostructures or complex nanostructured materials. This promises to reduce the time and cost required for their complete characterization.

\section{Supporting Information}

Supporting Information is available from the Wiley Online Library or from the author.

\section{Acknowledgements}

D.R. acknowledges support from the Royal Society's Newton International Fellowship scheme. P.A.M acknowledges financial support from the European Research Council under the European Union's Seventh Framework Programme (FP7/ 2007-2013)/ERC grant agreement 291522-3DIMAGE. P.A.M. also acknowledges financial support from the European Union's Seventh Framework Programme of the European Commission: ESTEEM2, contract number 312483. B.R.K. thanks the UK EPSRC for financial support (EP/J500380/1). 


\section{References}

[1] D. E. Newbury, Scanning 2005, 27, 227.

[2] H. S. Von Harrach, P. Dona, B. Freitag, H. Soltau, a Niculae, M. Rohde, J. Phys. Conf. Ser. 2010, 241, 012015.

[3] A. Gubbens, M. Barfels, C. Trevor, R. Twesten, P. Mooney, P. Thomas, N. Menon, B. Kraus, C. Mao, B. McGinn, Ultramicroscopy 2010, 110, 962.

[4] G. Kothleitner, W. Grogger, M. Dienstleder, F. Hofer, Microsc. Microanal. 2014, 20, 678.

[5] G. Haberfehlner, A. Orthacker, M. Albu, J. Li, G. Kothleitner, Nanoscale 2014, 6, 14563.

[6] J. M. Thomas, R. K. Leary, A. S. Eggeman, P. a. Midgley, Chem. Phys. Lett. 2015, DOI 10.1016/j.cplett.2015.04.048.

[7] C. Jutten, J. Herault, Signal Processing 1991, 24, 1.

[8] P. Kotula, M. Keenan, Microsc. Microanal. 2006, 538.

[9] G. Lucas, P. Burdet, M. Canto1ni, C. Hebert, Micron 2013, 52-53, 49.

[10] N. Bonnet, D. Nuzillard, Ultramicroscopy 2005, 102, 327.

[11] F. de la Peña, M.-H. Berger, J.-F. Hochepied, F. Dynys, O. Stephan, M. Walls, Ultramicroscopy 2011, 111, 169.

[12] D. Rossouw, P. Burdet, F. de la Peña, C. Ducati, B. R. Knappett, A. E. H. Wheatley, P. a. Midgley, Nano Lett. 2015, 15, 2716.

[13] D. Rossouw, R. Krakow, Z. Saghi, C. S. M. Yeoh, P. Burdet, R. K. Leary, F. de la Pena, C. Ducati, C. M. F. Rae, P. A. Midgley, Acta Mater. 2016, 107, 229.

[14] F. de la Peña, P. Burdet, T. Ostasevicius, M. Sarahan, M. Nord, V. T. F. J. Taillon, A. Eljarrat, S. Mazzucco, G. Donval, L. F. Zagonel, M. Walls, I. Iyengar, 2015, DOI 10.5281/zenodo. 27735 .

[15] E. Bingham, A. Hyvärinen, Int. J. Neural Syst. 2000, 10, 1.

[16] F. Pedregosa, G. Varoquaux, A. Gramfort, V. Michel, B. Thirion, O. Grisel, M. Blondel, P. Prettenhofer, R. Weiss, V. Dubourg, J. Vanderplas, A. Passos, D. Cournapeau, M. Brucher, M. Perrot, E. Duchesnay, J. Mach. Learn. Res. 2011, 12, 2825.

[17] B. R. Knappett, P. Abdulkin, E. Ringe, D. a Jefferson, S. Lozano-Perez, T. C. Rojas, A. Fernández, A. E. H. Wheatley, Nanoscale 2013, 5, 5765.

[18] C. C. Ahn, O. L. Krivanek, EELS Atlas, Gatan, 1983.

[19] C. Colliex, T. Manoubi, C. Ortiz, Phys. Rev. B 1991, 44.

[20] P. a Van Aken, B. Liebscher, V. J. Styrsa, Phys. Chem. Miner. 1998, 25, 323.

[21] K. M. Krishnan, Ultramicroscopy 1990, 32, 309.

[22] R. P. H. F. Auerhammer J., Ultramicroscopy 1989, 30, 365. 


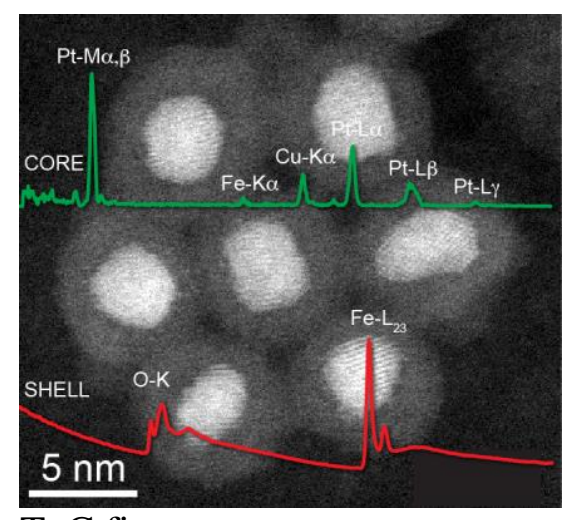

ToC figure

TOC text: A new method of determining the composition of spatially overlapping phases in a core-shell nanoparticle system is described. The technique involves the decomposition of simultaneously acquired micro-analytical x-ray (green) and electron energy loss signals (red) by independent component analysis, enabling the separation of spatially and spectrally overlapping signal components from both light and heavy elements. 